LA-UR- $98=1588$

Approved for public release; distribution is unlimited.

\author{
Title: \\ Femtosecond Scanning Tunneling \\ Microscope

\section{RECEIVED \\ OCT 051998 \\ OSTI} \\ Author(s): \\ Antoinette J. Taylor, \\ Giovanni P. Donati, \\ George Rodriguez, MST-11 \\ Timothy R. GosnelI, MST-10 \\ Stuart A. Trugman, T-11 \\ Daniel I. Some, Weizmann Institute \\ Submitted to: \\ DOE Office of Scientific and Technical \\ Information (OSTI)
}

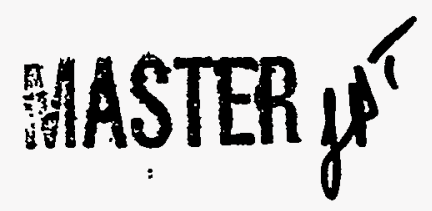

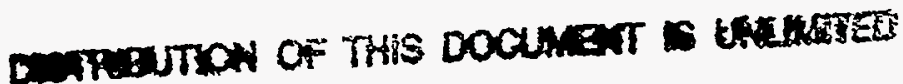

\section{Los Alamos}

NATIONALLABORATORY

Los Alamos National Laboratory, an affirmative action/equal opportunity employer, is operated by the University of California for the U.S. Department of Energy under contract W-7405-ENG-36. By acceptance of this article, the publisher recognizes that the U.S.

Government retains a nonexclusive, royalty-free license to publish or reproduce the published form of this contribution, or to aliow others to do so, for U.S. Government purposes. Los Alamos National Laboratory requests that the publisher identify this article as work performed under the auspices of the U.S. Department of Energy. The Los Alamos National Laboratory strongly supports academic freedom and a researcher's right to publish; as an institution, however, the Laboratory does not endorse the viewpoint of a publication or guarantee its technical correctness. 


\section{DISCLAIMER}

This report was prepared as an account of work sponsored by an agency of the United States Government. Neither the United States Government nor any agency thereof, nor any of their employees, makes any warranty, express or implied, or assumes any legal liability or responsibility for the accuracy, completeness, or usefulness of any information, apparatus, product, or process disclosed, or represents that its use would not infringe privately owned rights. Reference herein to any specific commercial product, process, or service by trade name, tradematk, manufacturer, or otherwise does not necessarily constitute or imply its endorsement, recommendation, or favoring by the United States Government or any agency thereof. The views and opinions of authors expressed herein do not necessarily state or reflect those of the United States Government or any agency thereof. 


\section{DISCLAIMER}

Portions of this document may be illegible in electronic image products. Images are produced from the best available original document. 


\title{
Femtosecond Scanning Tunneling Microscope
}

\author{
Antoinette J. Taylor,* Giovanni P. Donati, George Rodriguez (MST-11), \\ Timothy R. Gosnell (MST-10), \\ Stuart A. Trugman (T-11) \\ Daniel I. Some \\ Weizmann Institute
}

\begin{abstract}
This is the final report of a three-year, Laboratory Directed Research and Development (LDRD) project at the Los Alamos National Laboratory (LANL). By combining scanning tunneling microscopy with ultrafast optical techniques we have developed a novel tool to probe phenomena on atomic time and length scales. We have built and characterized an ultrafast scanning tunneling microscope in terms of temporal resolution, sensitivity and dynamic range. Using a novel photoconductive low-temperaturegrown GaAs tip, we have achieved a temporal resolution of 1.5 picoseconds and a spatial resolution of 10 nanometers. This scanning tunneling microscope has both cryogenic and ultra-high vacuum capabilities, enabling the study of a wide range of important scientific problems.
\end{abstract}

\section{Background and Research Objectives}

"The surface was invented by the devil," said physicist Wolfgang Pauli, referring to scientists' frustrated efforts to make quantitative the study of solid boundaries and interfaces. Pauli could hardly have predicted, however, the explosion of sophisticated techniques now routinely applied by the surface scientist to the investigation of structural order, stationary quantum states, and a zoo of dynamical processes that today make explicit Pauli's point. In the past few decades dozens of novel experimental techniques have helped expose the physics and chemistry of surfaces in new detail, but surface science unquestionably saw its first real revolution with the invention of the scanning tunneling microscope ${ }^{1}$ (STM) in 1982. As a 3-D imaging device with sub-angstrom spatial resolution, the STM created the first pictures of individual atoms; in so doing, our subjective mental images of atomic-scale surface structure were finally reconciled with objective observations. In the decade since its invention, tunneling microscopy has revealed incipient corrosion processes in iron metal, real-time dealloying of solid solutions in the

*Principal Investigator, e-mail: ttaylor@lanl.gov 
presence of acid-salt electrolytes, stacking effects of electro-deposited atoms around surface line defects, interface disorder between metal contacts and semiconductor devices, and realtime polymerization of single structural proteins. More exotic applications extend to investigations of chemical catalysis and micromechanics, and to the development of ultrahigh-density memories and medical implant technology.

The STM's sub-atomic spatial resolution is limited only by the extremely localized quantum tunneling of electrons between sample and probe. But its time resolution-and hence its ability to display surface dynamics as well as surface structure-is limited not by the 10 -femtosecond intrinsic time scale of the tunneling process itself, ${ }^{2}$ but by the $>1$-ms time-constants of the microscope's analog scanning and vertical-positioning electronics. For probing fast dynamics, time resolutions approaching $10 \mathrm{fs}$ are possible with ultrashortpulse lasers, but wave diffraction limits the spatial resolution of purely optical techniques to a few microns. During this project we explored our original ideas on how to combine tunneling microscopy with ultrafast laser methods in order to simultaneously probe physical phenomena on both atomic time as well as on atomic length scales.

Research in time-resolved scanning tunneling microscopy has been heating up. Over the time span of this project, three other groups have simultaneously begun to develop the field of picosecond scanning tunneling microscopy, with all three groups building viable femtosecond scanning tunneling microscopes (FSTM) based on photoconductively gating, on an ultrafast time scale, the current through the tip. ${ }^{3-5}$ Preliminary experiments with these instruments are currently being planned. As a result of the research performed during this project we have also concluded that the optimal architecture for an ultrafast STM consists of an STM whose tip is photoconductively gated. Our invention of a novel tip, which locates the ultrafast photoconductive switch at the tunneling tip, eliminates detrimental propagation effects previously present in this approach ${ }^{\mathrm{P} 4}$ We have also built our STM in a chamber with both ultra-high vacuum and cryogenic capabilities, ${ }^{\mathrm{P} 5}$ thus enabling a wide range of scientific studies with this novel tool.

\section{Importance to LANL's Science and Technology Base and National R\&D Needs}

Basic Scientific Impact: In physics, biology, materials science, and chemistry the techniques we have developed will make possible for the first time the study of realspace processes and excitations with combined femtosecond temporal resolution and atomic spatial resolution. It will become possible to spatially and temporally track phenomena such as vibronic motion, chemical reactions on surfaces, gap reduction in nonequilibrium superconductors, soliton conduction in molecular wires, and single-electron transfer in 
Coulomb blockade devices. Moreover, a local spatio/temporal probe such as the FSTM is essential for unraveling the physics of complex many-body materials systems. Although beyond the scope of this project, our demonstration of a femtosecond STM may prefigure the development of ultrafast temporal resolution in other scanning-probe microscopies such as atomic-force microscopy and near-field scanning optical microscopy. Moreover, an ultrafast spin-selective STM is the logical extension of our FSTM using the novel photoexcited-GaAs tip we have invented as an ultrafast source of spin-polarized electrons.

Applications in High-Speed Electronics Development: "Smaller, faster, cheaper," is the mantra of the integrated-electronics industry, yet today's standard techniques for diagnosing the performance of high-speed electronic and electro-optic devices face a technical discontinuity: On-wafer microwave probes give subpicosecond performance but they require a minimum contact-pad area of $10 \mu \mathrm{m}^{2}$. The bandwidth of an electro-optic sampling system can exceed $1 \mathrm{THz}$; however, the lateral resolution is limited to a few microns by the minimum focal-spot size. Although electron-beam techniques can yield high spatial resolution, such methods are practical only in vacuum and are deadly slow because space-charge effects limit beam blanking times. It gets worse. As an electronic device decreases in size, its surface area increases linearly in relation to its volume; hence the surface increasingly dominates the device's operation. None of the presently available techniques are both surface sensitive and surface selective, and none combine the requirements of ultrahigh speed and ultrahigh spatial resolution. Perhaps the most compelling technological application of the ultrafast STM is in the development of next-generation electronic devices embodying submicron spatial features and subpicosecond switching times.

An ultrafast STM can directly characterize the operation of submicron electronic and optoelectronic devices, providing critical feedback to device designers. Specific problems include:

- Electrical Contacts. In submicron devices the role of contacts, and hence of semiconductor-metal interfaces, is poorly understood. An ultrafast STM could reveal electric-field and voltage wavefront propagation around submicron electrical contacts. The contact structure might then be redesigned to minimize wavefront distortions. A second problem: Because small contacts necessarily support high current densities, enhanced electromigration of aluminum grains degrades the performance of submicron devices. There is currently no data on the dynamics of this detrimental process.

- Electrical Crosstalk. Signal crosstalk in passive device interconnects, between adjacent active devices on a high-density chip, and within a submicron device itself is a nascent 
problem in microelectronic-device design. Ultrafast tunneling microscopy can detect the presence of crosstalk and measure distortion of electrical waveforms as a function of position within the device under test. As before, redesign of the electronic device would compensate performance losses, but one can equally well imagine the development of predictive models for device interactions benchmarked by ultrafast STM measurements.

- Transport Processes. More fundamental aspects of submicron device performance can be resolved with ultrafast tunneling microscopy. Of specific concern is the physics of charge-carrier transport through thin films and across interfaces. The ultrafast STM might be used to inject ballistic electrons or holes into a sample heterostructure; ultrafast pump-probe measurements of the resulting current flow would isolate for the first time transport phenomena initiated by unthermalized charge carriers. Since the STM's dc bias voltage can control the energy of the injected carriers, a wealth of spectroscopic information can be obtained by tuning of the carrier energy, especially through the critical ballistic regime. This ultrafast variation of ballistic-electron emission spectroscopy ${ }^{6}$ would render unique time-resolved data on carrier scattering in electronic materials. Such fundamental but heretofore elusive quantities as the transient carrier mobility and its relaxation rate could be determined as a function of the carrier excitation energy.

- New Materials. Transport in novel electronic materials could be investigated with our techniques. A critical experimental feature intrinsic to the ultrafast STM and relevant to all of the above applications is that problematic high-speed electrical contacts are never needed. The techniques can therefore be just as easily applied to conventional semiconductors as to more exotic materials such as photoconducting polymers, fullerenes, and quasi-one-dimensional MX electronic materials.

- Nanometer Devices. Conventional semiclassical device physics breaks down in electronic structures with nanometer spatial features. The advance of "nanotechnology" for applications in ultrahigh speed, ultracompact electronics therefore proffers the triple burden of developing new theories of operation, new fabrication techniques, and new diagnostic methods that fully incorporate quantum mechanical effects at the outset. Despite these daunting challenges, research groups both in the US and abroad are vigorously investigating experimental and theoretical concepts for electronic nanodevices that may in the next century foment the most significant structural change in the electronics industry since the invention of the transistor. Since conventional scanningprobe microscopies have already proven themselves the tools of choice in this new field 
of research, the creation and characterization of real devices will mandate the extension of these techniques into the femtosecond time domain.

Importance to LANL's Science and Technology Base: Our femtosecond scanning tunneling microscope will have a wide variety of applications within LANL. The development of this instrument advances the forefront of materials science at the Laboratory and strengthens the Laboratory's technology base in the core competency of advanced and nuclear materials. The following potential classes of experiments for the FSTM are of interest to researchers in technical divisions spanning the Laboratory:

- carrier dynamics in metals and semiconductors,

- quasi-particle dynamics in complex materials (cuprates, manganates, potentially even plutonium) where local structure plays an important role in the physics of the interactions,

- vortex dynamics in high- $\mathrm{T}_{\mathrm{C}}$ superconductors,

- dynamics of optically driven, coherent phonon oscillations of adsorbed molecules on self-assembled monolayers,

- nanocrystal photodynamics, and

- dynamics of charge density waves.

\section{Scientific Approach and Accomplishments}

\section{Overview}

By combining scanning tunneling microscopy with ultrafast optical techniques we have developed a novel tool to probe physical phenomena on both atomic time and atomic length scales. The salient goal of this project was the construction of the first ultrafast scanning tunneling microscope with a temporal resolution of $1 \mathrm{ps}$ or less. We adopted a two-generation R\&D approach for the development of the FSTM. We built the first generation FSTM, which consisted of integrating an ambient STM with a femtosecond laser system and customizing this system as a flexible ultrafast pump-probe set-up. We then used this first generation system to implement and evaluate different strategies for obtaining an ultrafast tunneling signal. In particular, we examined three methods for demonstrating ultrafast time resolution of tunneling currents: 1 ) direct optical rectification of the femtoseocnd laser pulse, 2) the application of an ultrafast bias using a novel near-field terahertz emitter, and 3) the incorporation of a photoconductive switch in the STM circuit.

Based on our experimental studies of the three techniques, we chose the use of a photoconductive switch for out second generation FSTM since its use resulted in the most robust performance of the FSTM. We then optimized the performance of this tunneling scheme. Since many applications of an ultrafast STM to interesting problems in 
fundamental science require both very clean surfaces as well as low temperatures to prevent the rapid dephasing of photoexcitations, we built a second generation instrument: an ultrahigh vacuum, variable temperature (cryogenic) FSTM using the photoconductive switch to obtain picosecond resolution. We expect this second generation instrument to enable the study of a wide range of important scientific problems.

Throughout this project the performance of our FSTM was investigated and benchmarked using picosecond electrical transients generated by the pump beam on an optoelectronically switched, coplanar stripline (sketched in Figure 1) as follows: The coplanar stripline consists of 50- $\mu \mathrm{m}$ wide, $0.1-\mu \mathrm{m}$ thick, 6 -mm long gold lines deposited $10-\mu \mathrm{m}$ apart on a silicon-on-sapphire (SOS) substrate with a voltage drop of $25 \mathrm{~V}$ across the lines. A $10-\mu \mathrm{m}$ by $10-\mu \mathrm{m}$ radiation-damaged SOS switch with a carrier lifetime of about $1 \mathrm{ps}$ is fabricated between the striplines. When the ultrafast pump pulse is focused onto this radiation-damaged SOS switch, an ultrafast electrical pulse is generated that propagates down the stripline. (The SOS is radiation damaged with a dose of $10^{15}$ ions $/ \mathrm{cm}^{2}$ of $150-\mathrm{keV}$ singly ionized oxygen atoms at a temperature of $0{ }^{\circ} \mathrm{C}$ to reduce the carrier lifetime to 1-2 ps.) Using optoelectronic correlation techniques ${ }^{7}$ in conjunction with a second radiation-damaged SOS switch fabricated to the side of one stripline, the resultant correlation signal (shown in Figure 2) indicates that the deconvolved temporal duration of this electrical pulse is approximately $2.0 \mathrm{ps}$. The second pulse in the figure is the reflection of the launched pulse off the end of the stripline.

The same ultrafast optical pump/probe setup is used throughout this project: A mode-locked Ti:sapphire laser provides $100-\mathrm{fs}, 800-\mathrm{nm}$ optical pulses at a repetition rate of $82 \mathrm{MHz}$. Signal-to-noise considerations dictate the use of such a high duty-cycle, but low-energy ( $5 \mathrm{~nJ} / \mathrm{pulse}$ ) laser source. The output from this laser is split into a pump beam and a time-delayed probe beam. The pump beam generates picosecond-duration voltage transients which propagate on a coplanar stripline. The probe beam traverses a scanning delay line producing a variable delay $\tau$ between the pump and probe pulses and then is used to gate resultant tunneling transient produced by the pump beam. A more general ultrafast STM experiment would consist of triggering an optical, chemical, electrical, or magnetic transient on the surface of interest with the ultrashort pump pulse while gating the tunnel current at an adjustable time delay with the ultrashort probe pulse. Data captured with a sequence of time delays stroboscopically represents the fully evolved femtosecond dynamics of the system under study. 


\section{First Generation FSTM}

The first generation FSTM consists of a NanoScope III STM integrated with the ultrafast pump-probe setup described earlier and with stabilization and data acquisition customized for femtosecond pump/probe experiments. With this system we investigated the three strategies for obtaining an ultrafast tunneling signal: optical rectification via the tipsample nonlinearity, terahertz biasing of the tip-sample junction, and photoconductive gating of the tunneling current from the tip.

We first describe our work on ultrafast gating of the transient tunneling current using optical rectification of the femtosecond probe pulse. The concept for this approach is as follows: In its basic operation, an STM functions as a point-contact tunnel diode with the air or vacuum gap between the sample surface and the probe serving as the insulating barrier. A dc bias voltage of about $100 \mathrm{mV}$ applied to the diode encourages the continuous flow of a tunnel current through the insulating layer. The tip-to-surface distance is typically $10 \AA$; hence the electric field within the barrier is about $1 \mathrm{MV} / \mathrm{cm}$. Since the electric field of even a low-energy but focused femtosecond laser pulse can be easily made greater than 5 $\mathrm{MV} / \mathrm{cm}$, such a pulse propagating between the STM probe and the sample surface can significantly modify the instantaneous tunnel current. Integrated over the full duration of the pulse, a nonzero change is obtained in the average tunnel current because of the nonlinear $I-V$ characteristic of the junction: $I=I_{L}\left(V+\beta V^{3}\right)$ for a metal-insulator-metal junction, ${ }^{8}$ where $\mathrm{I}_{\mathrm{L}}$ and $\beta$ quantify, respectively, the linear and nonlinear responses of the junction. In other words, the tunnel junction rectifies the optical pulse to give an observable change in the tunnel current. This approach is both simple in its implementation and is potentially noninvasive. Also, $10 \mathrm{fs}$ temporal resolution could potentially be achieved, limited by the duration of the laser pulse (which could be $<10 \mathrm{fs}$ ) and by the intrinsic time scale of the tunneling process (which should also be $<10 \mathrm{fs}$ ) 2 .

Unfortunately, at visible wavelengths optical rectification is masked by thermal electron emission and multiphoton electron emission for high repetition rate (100 MHz), low-energy (nJ) probe pulses such as used here. If high energy $(\mathrm{mJ})$, low repetition rate $(10 \mathrm{~Hz})$ sources are used, multiphoton emission only dominates over optical rectification. Under ultra-high vacuum conditions a transient signal is present, ${ }^{9}$ but since it is due to multiphoton-induced electron emission and not tunneling, it does not yield the desired atomic spatial resolution.

As the energy of the probe photon is decreased, we expect multiphoton and thermal electron emission to interfere less with the tunneling signal. The extreme extension of this concept captures our second approach to the FSTM: Instead of directly applying the optical 
pulse to the STM's sample-tip interface, a single cycle of far-infrared or terahertz

bandwidth radiation can be generated by optical rectification of a femtosecond laser pulse in an ancillary nonlinear material.10,P1 The freely propagating terahertz pulse produced by this means is then focused onto the STM junction to give a unipolar modification to the electric field and hence to the tunnel current. Minimum measured terahertz pulse widths are $30 \mathrm{fs}$, and should be theoretically limited only by the duration of the input optical pulse. In the context of STM experiments, this method's potential advantages include improved signal-to-noise ratio and reduced interference with the dynamics under study and the tipsample interaction. Moreover, a temporal resolution approaching $30 \mathrm{fs}$ should be achievable.

The application of an ultrafast bias ${ }^{\mathrm{P} 2}$ using a novel near-field biased terahertz emitter was investigated. Normally, terahertz radiation can only be focused down to the diffraction limit, which is of the order $1 \mathrm{~mm}$. In order to increase the field strength of the terahertz pulses produced with the low laser power but high duty cycle present in our system, we developed a novel near-field terahertz emitter: A $100 \mu \mathrm{m}$ photoconductive antenna on a GaAs chip measuring $150 \times 150 \mu \mathrm{m}^{2}$ and only $30 \mu \mathrm{m}$ thick was fabricated and placed at the end of an optical fiber, with leads attached for application of a bias. This emitter could be positioned to within $\sim 100 \mu \mathrm{m}$ from the tunneling junction, so that the terahertz radiation cannot diffract by more than about $100 \mu \mathrm{m}$, hence reducing the spot size and increasing the transient electric field by an order of magnitude. We estimate that the radiated field, picked up by the STM junction is $5-50 \mathrm{mV}$, which should produce a transient tunneling current at the lower end of our instrument sensitivity. Although this approach seems promising, the relatively short lifetime (several hours) of these near field emitters yielded this approach unsuitable for as complex an instrument as the FSTM. However, if this lifetime issue were to be resolved, this approach could become viable.

For our third approach we introduce a nonlinearity to the tip assembly, separate from the tunnel junction. More specifically, an ultrafast photoconductive switch is integrated into the STM tip assembly to provide a gating nonlinearity. The pump laser generates a transient response on the sample, which creates a transient tunneling current. The probe laser activates the switch, which selectively enhances a temporal slice of the tunneling current. As the time delay between the pump and the probe is varied, the temporal evolution of the sample response to the pump is mapped out. The temporal resolution of this technique is limited by the temporal width of the response of the photoconductive switch, typically about $1 \mathrm{ps}$. This approach has the advantage that the 
ultrafast instrument response is decoupled from the tip-sample nonlinearity. Therefore, this approach can be used to study any laser-generated excitation on the sample, as well as study the intrinsic nonlinearity of the STM tunnel junction itself.

The tunneling tip is attached to a coplanar stripline with an integrated photoconductive switch. The tip consists of 0.001 -inch-diameter tungsten wire about 0.1 $\mathrm{mm}$ long glued to the photoswitch using conductive epoxy. The photoswitch consists of two coplanar gold transmission lines $0.05 \mathrm{~mm}$ wide by $0.1 \mathrm{~mm}$ long deposited $0.05 \mathrm{~mm}$ apart on a low-temperature-grown GaAs (LT-GaAs) substrate. LT-GaAs serves as the ultrafast photoconductive material since it has a lifetime of about $1 \mathrm{ps}$. Using this tip we obtain a time resolution of $2.5 \mathrm{ps}$ with a few tens of picoamps of average transient tunneling current. The performance of the STM is illustrated with the tunneling waveforms shown in Figure 3. Figure 3a reveals the transient tunneling signal when the tip is in contact with the sample. In this mode, the FSTM acts as a simple voltage probe that photoconductively measures the voltage waveform on the stripline. In Figure $3 b$, the tip has been lifted off the sample, so that it is in weak contact, resulting in a total resistance of the switch and the tunnel junction of $10 \mathrm{M} \Omega$. For the waveform in Figure $3 \mathrm{c}$ the tip resistance has been increased to $16 \mathrm{M} \Omega$ and the resultant signal is a true tunneling signal. For this circuit configuration of the tip and photoconductive switch, the transient current corresponding to the tunneling signal is expected to be the derivative of the signal obtained with the tip in contact with the sample, as seen in Figure 3c. Figure $3 \mathrm{~d}$ shows how the waveform changes as the tip is removed further from the sample. For a total resistance of $33 \mathrm{M} \Omega$, the signal retains its derivative shape, but decreases in magnitude.

Since the use of the photoconductive switch produces the best transient tunneling signal, we decided to adopt and optimize this approach for our second generation FSTM. To this end we have developed a novel STM tip consisting of a cleaved GaAs substrate with a 1-mm thick epilayer of low-temperature-grown GaAs (LT-GaAs) deposited on the face. Since the LT-GaAs has a carrier lifetime of $1 \mathrm{ps}$, photoexcitation of the tip with an ultrafast, above-bandgap pulse both provides carriers for the tunneling current and photoconductively gates (with ps resolution) the current from the tip. With this tip we have demonstrated a temporal resolution of $1.5 \mathrm{ps}$ in tunneling mode. ${ }^{\mathrm{P4}}$

Previously, tips used for a photoconductively gated STM have consisted of a piece of metal wire (typically $\mathrm{Pt}$ ) in series with a photoconductive switch fabricated from either radiation-damaged SOS or LT-GaAs., 11 Recently, a new optoelectronic tip was fabricated using photolithographic techniques where the LT-GaAs optoelectronic switch was located 
$30 \mu \mathrm{m}$ from the switch, minimizing the effects of signal damping and dispersion. ${ }^{12}$ Our tip effectively locates the ultrafast switch at the tunneling tip, eliminating all detrimental propagation effects. It is an elegant solution that is simple and cheap to fabricate. The properties of photoexcited GaAs STM tips have previously been studied, 13,14 however, the combination of an LT-GaAs tip with ultrafast optical excitation to yield ps temporal resolution has not previously been demonstrated.

A sketch of the LT-GaAs STM tip is shown in Figure 4. The tip consists of a (100) GaAs substrate cleaved to a size of $0.1 \mathrm{~mm}$ by $0.1 \mathrm{~mm}$ by $0.05-\mathrm{mm}$ thick and covered with a 1-mm-thick epilayer of LT-GaAs. The LT-GaAs layer is grown at $250^{\circ} \mathrm{C}$ and subsequently annealed for thirty minutes at $550^{\circ} \mathrm{C}$, yielding an approximate carrier lifetime of $1 \mathrm{ps}$. The probe beam is focused on the LT-GaAs face at about 20 degrees off normal incidence. This GaAs tip is bonded to 0.01 -inch-diameter tungsten wire using a gold contact pad ( $50 \mu \mathrm{m}$ by $50 \mu \mathrm{m}$ by $0.1-\mu \mathrm{m}$-thick). The dark resistance of the tip is typically

3-5 G $\Omega$. We emphasize the simplicity of these tips, which makes their fabrication easy and cheap.

\section{Second Generation FSTM}

Our second generation FSTM ${ }^{\mathrm{P} 5}$ is based on an RHK STM-1000 head with a threepoint piezo-controlled manipulator and associated control electronics. The head is mounted in vacuum chamber with multi-port optical access and $10^{-10}$ Torr base pressure. The sample is mounted on a platform that can be heated and cooled to cryogenic temperatures. Our LT-GaAs tip is routinely incorporated in this set-up, although a standard metal tip (with or without a photoconductive switch) can also be used.

The detailed experimental set-up for the FSTM is shown in Figure 5. A commercial mode-locked Ti:sapphire laser provides $100-\mathrm{fs}, 800-\mathrm{nm}$ optical pulses at a repetition rate of $82 \mathrm{MHz}$. The output from this laser is split into a pump beam and a time-delayed probe beam. The pump beam is mechanically chopped at a frequency of $2 \mathrm{kHz}$ and used to generate picosecond-duration voltage transients that propagate on a coplanar stripline. The probe beam traverses a scanning delay line producing a variable delay $\tau$ between the pump and probe pulses and then is focused using a $\mathrm{mm}$ focal length lens onto the face of a LTGaAs STM tip mounted above the stripline. In this configuration the probe both provides carriers for the tunneling current and photoconductively gates the current from the tip. The tip is mounted in a RHK STM- 1000 piezo-controlled head which is stabilized with its own control electronics and provides a DC bias to the stripline of typically 1-7 V. The tunneling current from the tip is amplified by a $100 \mathrm{mV} / \mathrm{nA}$ current-to-voltage converter connected to 
both via a buffered output to a lock-in amplifier. The feedback loop changes the distance of the tip to the stripline via the piezo-controlled head to maintain a constant current. The output of the lock-in amplifier reveals the transient tunneling signal modulated at the $2-\mathrm{kHz}$ chopping frequency, which is recorded for each value of delay $\tau$.

When this tip is illuminated with up to $30 \mathrm{~mW}$ of optical power we are able to demonstrate atomic resolution using a highly oriented pyrolytic graphite sample. Representative I-V curves for the tip in contact with the stripline for several photoexcitation powers is shown in Figure 6. The photovoltage, determined by the current null is $0.6 \mathrm{~V}$, independent of optical excitation power. The photoinduced current (current at zero bias) depends on optical power and is $0.2 \mathrm{nA}$ for an incident optical power of $29 \mathrm{~mW}$.

The ultrafast transient characteristics of the STM tip are demonstrated in Figures 7 and 8. The linear dependence of peak transient current with optical power measured with the tip in contact with the stripline is demonstrated in Figure 7. When the tip is lifted off the stripline, placed in tunneling mode, and the feedback loop is engaged, the feedback loop corrects for changes in optical power and the peak tunneling current remains constant as the probe power changes. The inset in Figure 7 reveals the current waveforms detected when the tip is in contact and tunneling $(R=0.3 \mathrm{G} \Omega)$. Unlike previously obtained results with metal tips, ${ }^{3-5}$ the waveforms in contact and tunneling mode are remarkably similar with the GaAs tip. More specifically, the signal in tunneling mode is not the derivative of the signal in contact mode. In Figure 8 we plot the peak transient tunneling current as a function of tunneling gap resistance. (All data points represent scans in tunneling, rather than contact mode.) The peak transient tunneling current decreases linearly with gap resistance out to $10 \mathrm{G} \Omega$ and then decreases more slowly out to $100 \mathrm{G} \Omega$ where it reaches our noise level. The corresponding waveforms shown in the inset show only slight shape changes with gap resistance and reveal a full width at half maximum of $2.5 \mathrm{ps}$.

Deconvolving the 2 ps pulsewidth of the electrical pulse on the stripline yields a $1.5 \mathrm{ps}$ temporal resolution for the STM.

In Figure 9 we demonstrate the ability to measure a temporal and spatial image of picosecond electrical waveform propagating along the stripline with our GaAs tip. These measurements, taken with the tip in contact mode, show the picosecond voltage pulse and its reflection off the end of the stripline as a function of distance down the stripline. As the end of the stripline is approached the two peaks coalesce into one.

Figure 10 demonstrates our ability to take "nanofast movies" with our FSTM. Figures 10a, 10b, and 10c are three atomic scale images of the stripline over the same 
$10,000 \mathrm{~nm}^{2}$ area taken at time delays given in the waveform shown in Figure $10 \mathrm{~d}[-10 \mathrm{ps}$ before the voltage transient (10a), at the peak of the voltage transient (10b), and $6 \mathrm{ps}$ after the voltage transient (10c)]. Features $10 \mathrm{~nm}$ or less in diameter, which presumably correspond to defects responding to the transient voltage, appear and disappear reproducibly in these three sequential picosecond-duration timeframes. Thus, for the first time, we have achieved a simultaneous spatial resolution of $10 \mathrm{~nm}$ and a temporal resolution of $1.5 \mathrm{ps}$.

\section{Conclusion}

In conclusion, we have successfully combined scanning tunneling microscopy with ultrafast optical techniques and developed a novel tool to probe phenomena on atomic time and length scales. We have built and characterized our femtosecond STM in terms of temporal resolution, sensitivity and dynamic range. Using a novel photoconductive lowtemperature-grown GaAs tip, we have achieved a temporal resolution of $1.5 \mathrm{ps}$ and a spatial resolution of $10 \mathrm{~nm}$. This FSTM has both cryogenic and ultra-high vacuum capabilities, enabling the study of a wide range of important scientific problems.

\section{Publications}

1. Rodriguez, G. and Taylor, A. "Screening of the bias field in terahertz generation from photoconductors, Opt. Lett. 21, (1996). LAUR-96-3490

2. Taylor, A., Rodriguez, G., and Some, D., "Ultrafast field dynamics in photoconductors," Opt. Lett. 22, 715 (1996). LAUR-96-3535

3. Some, D. and Nurmikko, A., "Ultrafast Photoexcited Cyclotron Emission: Contibutions from Real to Virtual Excitations," Phys. Rev. B 53, R13295-R13298 (1996). LAUR-95-3563

4. Donati, G. Some, D., Rodriguez, G., and Taylor, A., "Ultrafast scanning tunneling microscopy using a photoexcited low-temperature-grown GaAs tip, submitted to Appl. Phys. Lett. (1997). LAUR-97-3900

5. Donati, G., Some, D., and Taylor, A., "An ultrahigh vacuum, cryogenic femtosecond scanning tunneling microscope," in preparation. 


\section{References}

[1] Binnig, G. and Rohrer, H., "Scanning tunneling microscopy," Helv. Phys. Acta 55, 726-735 (1982).

[2] Cutler, P., Feuchtwang, T., Tsong, T., Nguyen, H., and Lucas, A., "Proposed use of a scanning-tunneling-microscope tunnel junction for the measurement of a tunneling time," Phys. Rev. B 35, 7774-7775 (1987).

[3] Weiss, S., Ogletree, D., Botkin, D, Salmeron, M., and Chemla, D., "Ultrafast scanning probe microscopy," Appl. Phys. Lett. 63, 2567-2569 (1993).

[4] Groenveld, R. and van Kempen, H., "The capacitive origin of picosecond electrical transients detected by a photoconductively gated scanning tunneling microscope," Appl. Phys. Lett. 69, 2294-2296 (1996).

[5] Keil, U., Jensen, J., and J. M. Hvam, J., "Fiber coupled ultrafast scanning tunneling microscope," J. Appl. Phys. 81, 2929-2934 (1997).

[6] Kaiser, W. and Bell, L., " Direct investigation of subsurface interface electronic structure by ballistic-electron-emission microscopy," Phys. Rev. Lett. 60, 1406-1409 (1988).

[7] Smith, P., Auston, D., and Nuss, M., "Subpicosecond photoconducting dipole antennas," IEEE J. Quantum Electronics 24, 255-260 (1988).

[8] Simmons, J., "Generalized formula for the electric tunnel effect between similar electrodes separated by a thin insulating film," J. Appl. Phys. 34, 1793 (1963).

[9] Pfeiffer, W., Sattler, F. Vogler, S., Gerber, G., Grand, J.-Y., and Moller, R., "Photoelectron emission in femtosecond laser assited scanning tunneling microscopy," Appl. Phys. B 64, 265-268 (1997).

[10] Wu, Q. and Zhang, X.-C., "Free space elctro-optic smapling of mid-infrared pulses," Appl. Phys. Lett. 71, 1285-1286 (1997).

[11] Botkin, D., Glass, J., Chemla, D., Ogletree, D., Salmeron, M., and Weiss, S., "Advances in ultrafast scanning tunneling microscopy," Appl. Phys. Lett. 69, 1321-1323 (1996).

[12] Groeneveld, R., Rasing, Th., Kaufmann, L., Smalbrugge, E., Wolter, J., Melloch, M., and van Kempen, H., "New optoelectronic tip design for ultrafast scanning tunneling microscopy," J. Vac. Sci. Technol. B 14, 861 (1996)

[13] Prins, M., van der Wielen, M., Jansen, R., Abraham, D., and van Kempen, H., "Photoamperic probes in scanning tunneling microscopy," Appl. Phys. Lett. 64, 12071209 (1994).

[14] Prins, M., Jansen, R., Groeneveld, R., van Gelder, A. and van Kempen, H., "Photoelectrical properties of semiconductor tips in scanning tunneling microscopy," Phys. Rev. B 53, 8090-8113 (1996). 


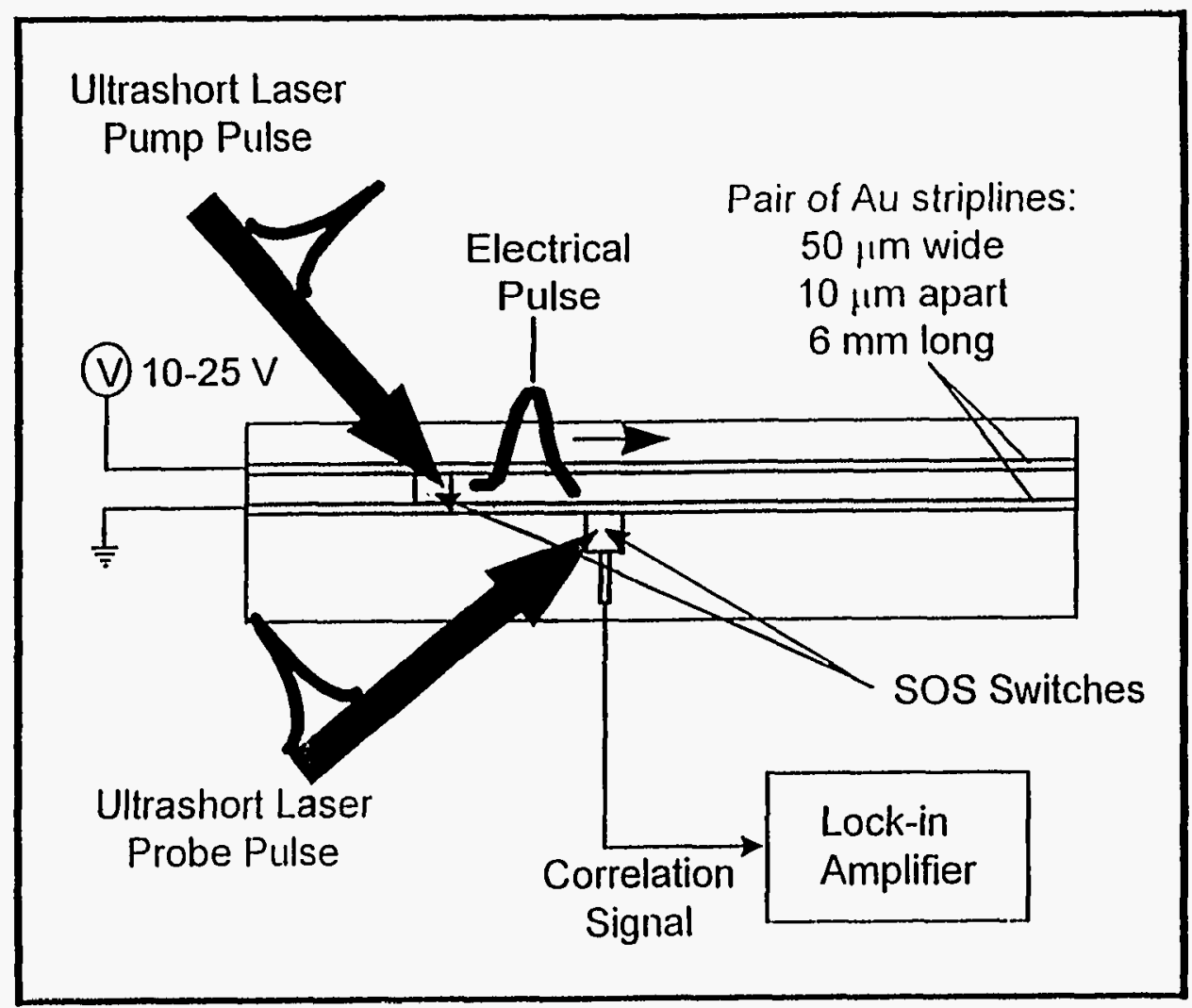

Fig. 1. Schematic of optoelectronically-switched coplanart stripline used to generate picosecond electrical transients to benchmark the performance of the FSTM. 


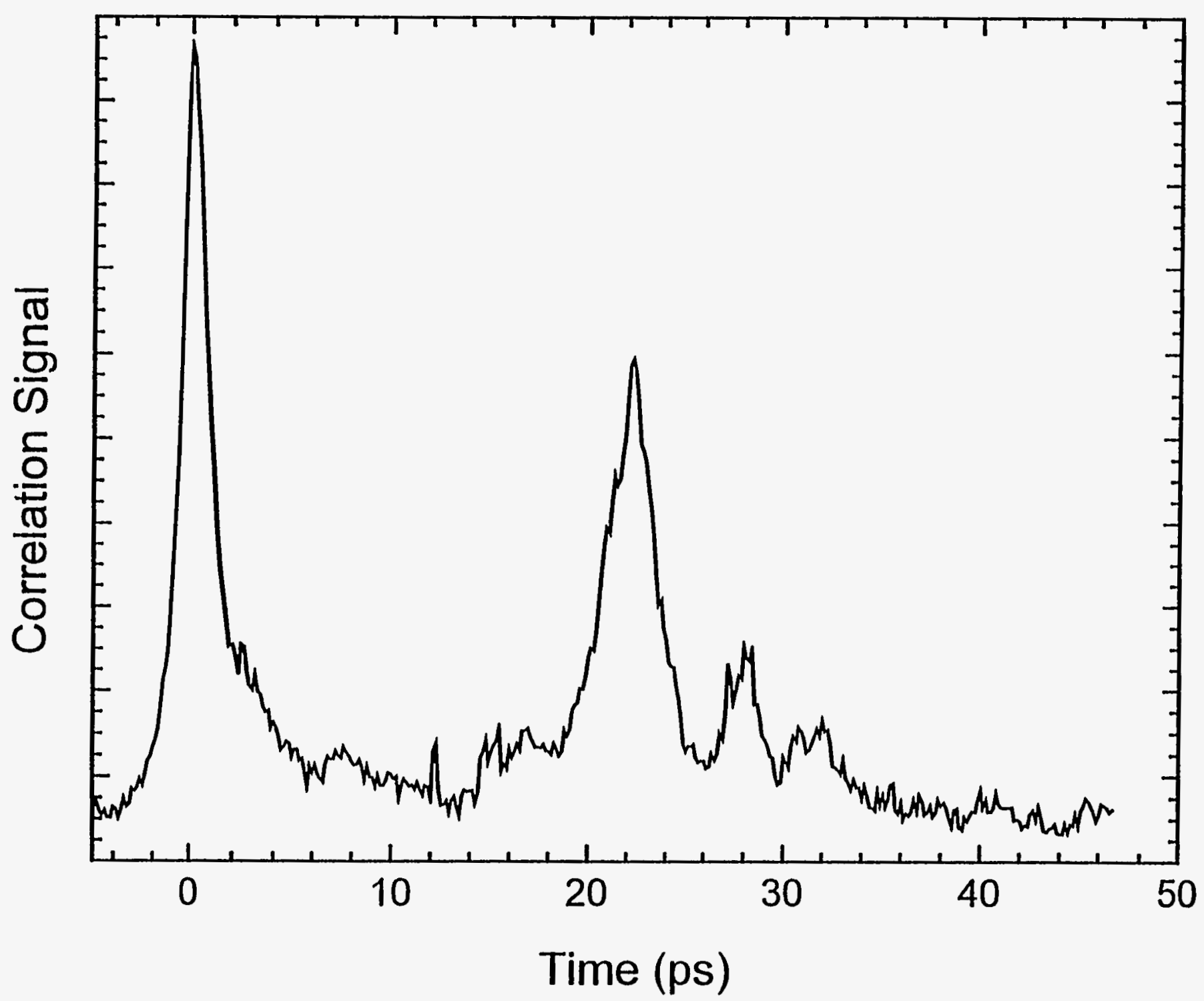

Fig. 2. Correlation signal from the stripline shown in Figure 1 when excited and sampled by $100-\mathrm{fs}, 800-\mathrm{nm}$ optical pulses. The deconvolved temporal duration of the electrical pulse is 2 ps. The second pulse is the reflection of the launched pulse off the end of the 


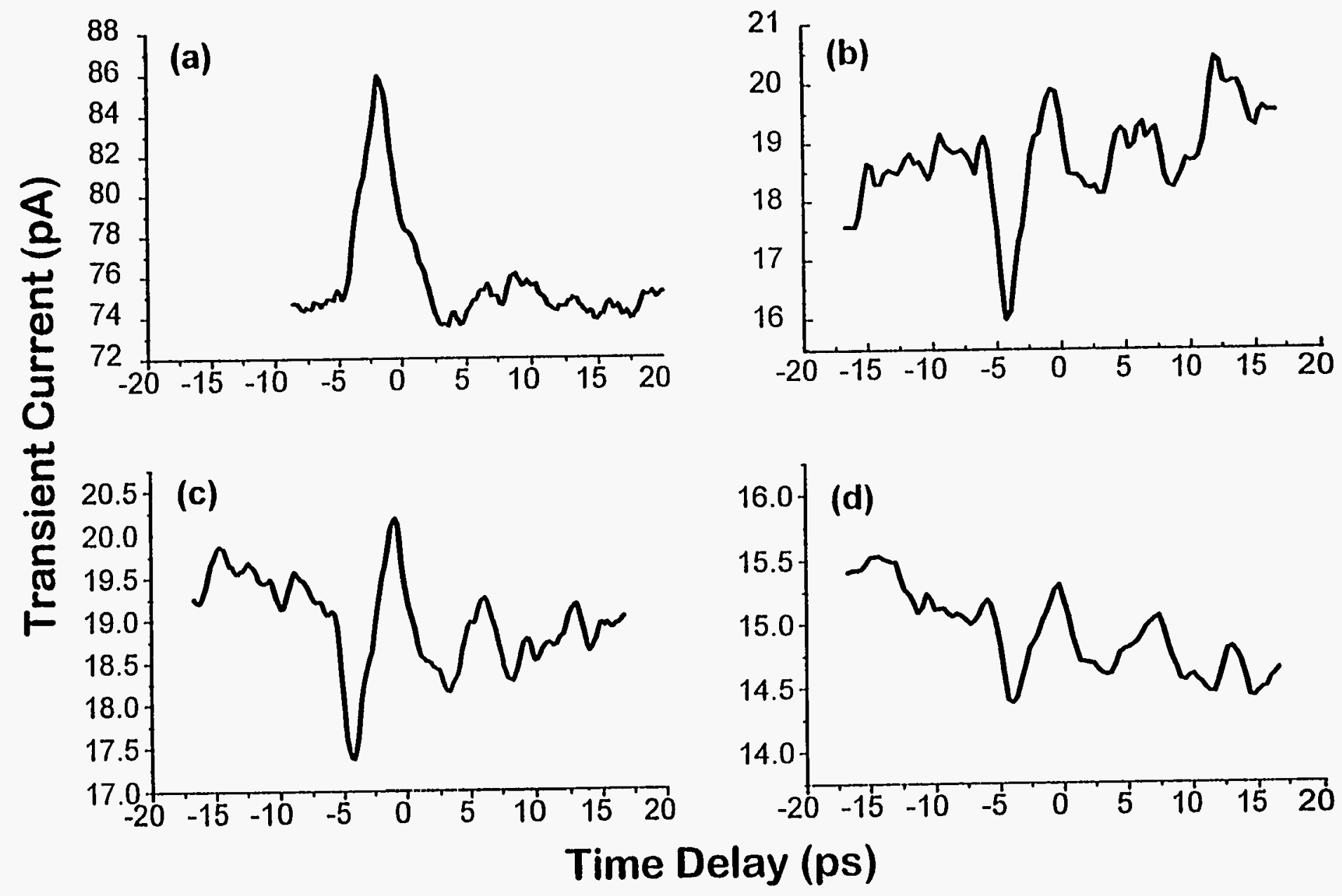

Fig. 3. Ultrafast waveforms from the FSTM using a metal tip in series with a photoconductive switch: (a) Tip in contact with sample. (b) Tip weakly contacting sample with a total resistance of $10 \mathrm{M} \Omega$. (c) True tunneling signal with a total resistance of $16 \mathrm{M} \Omega$.

(d) True tunneling signal with a total resistance of $33 \mathrm{M} \Omega$. 


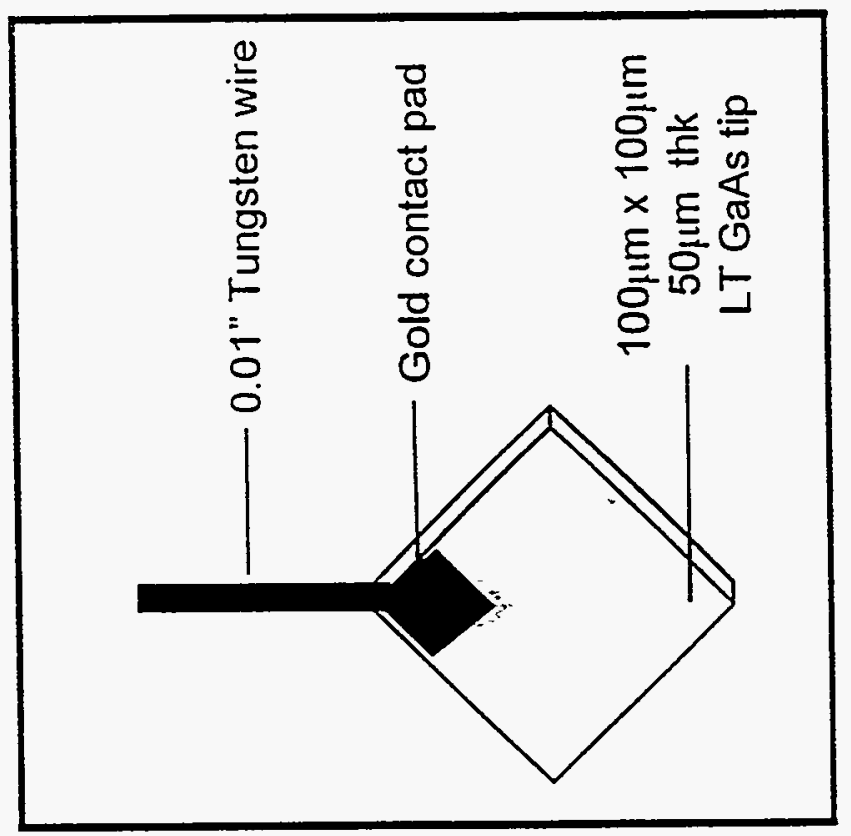

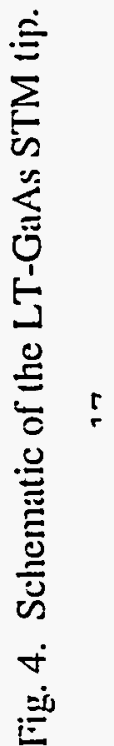



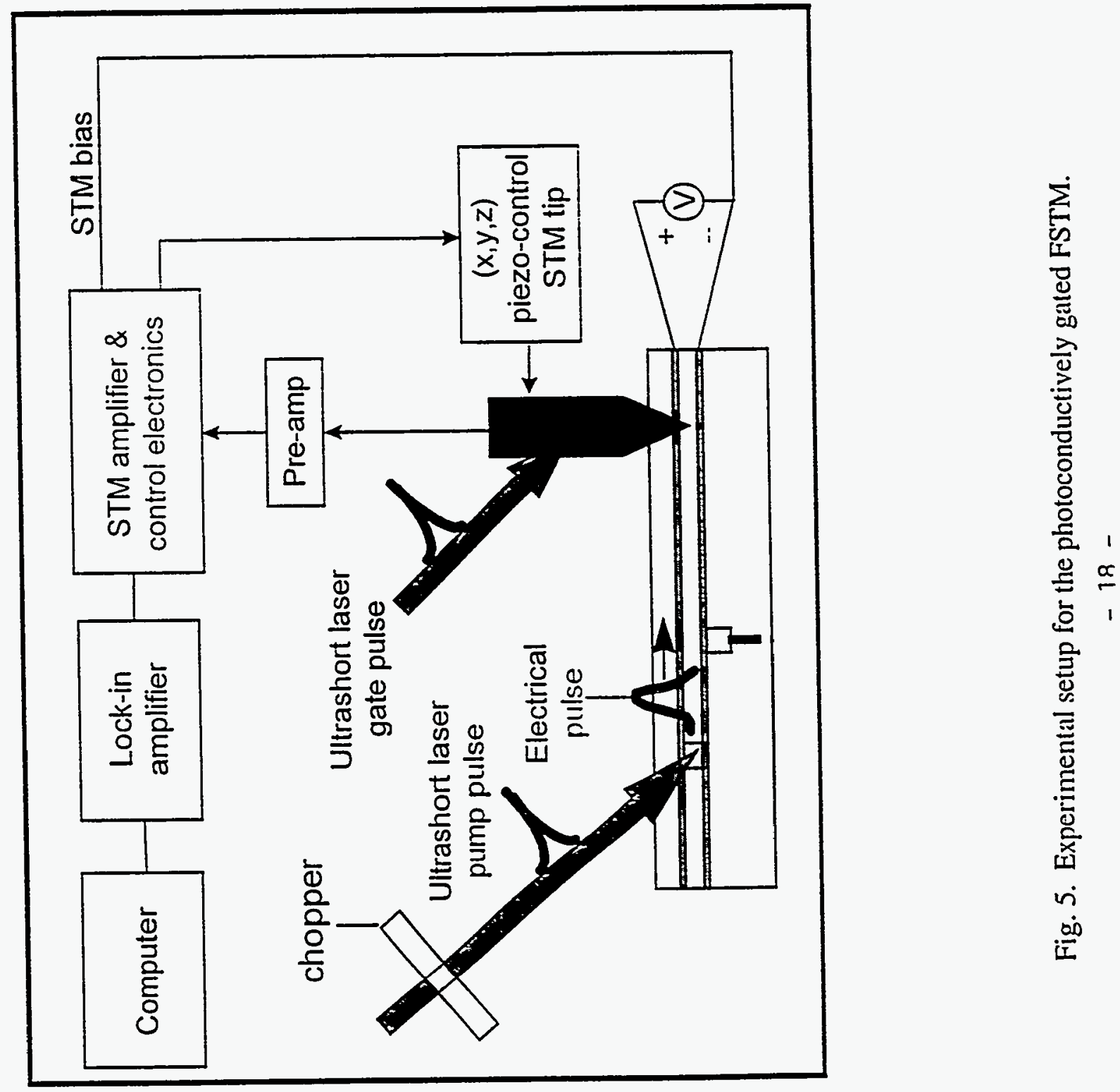


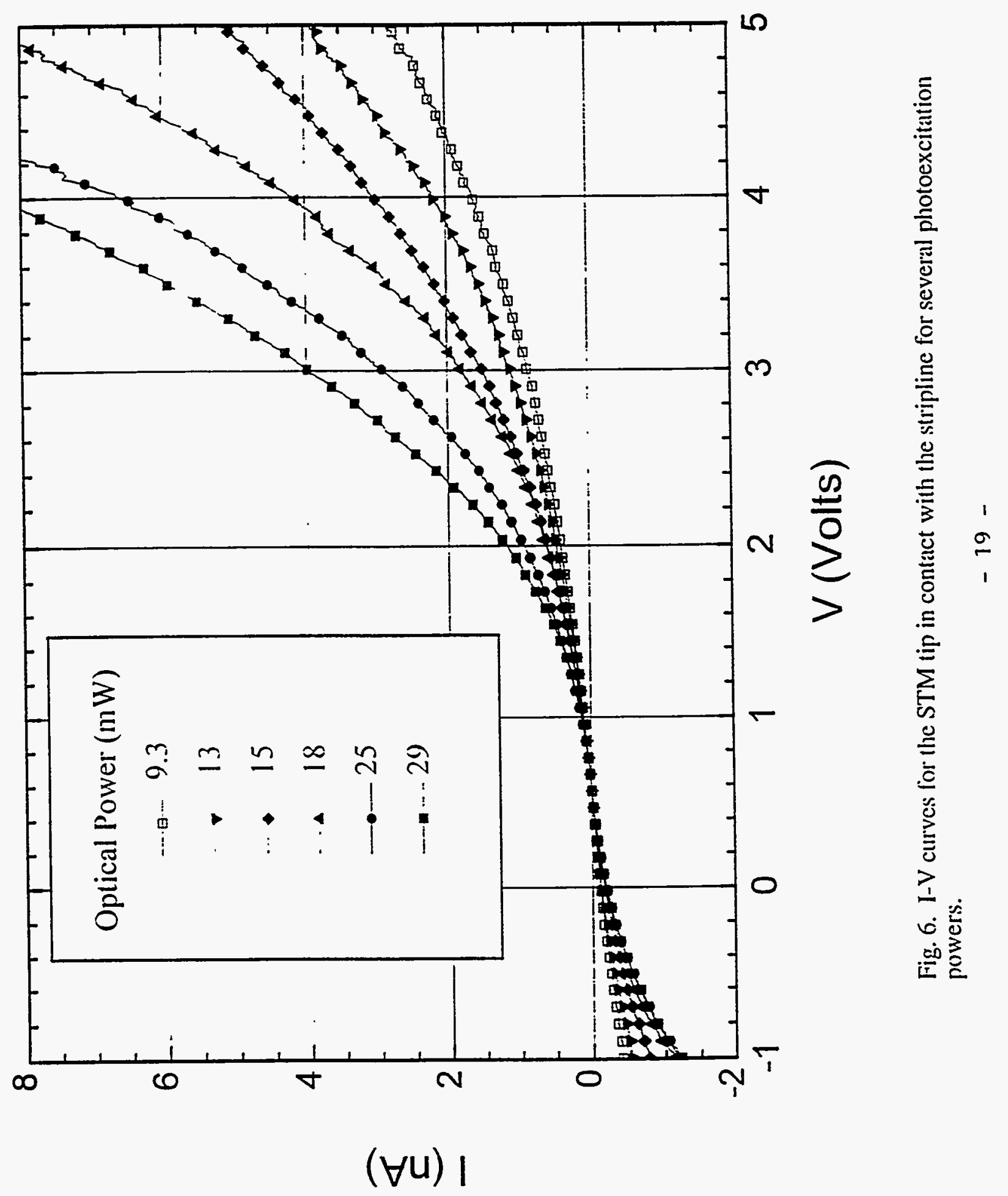




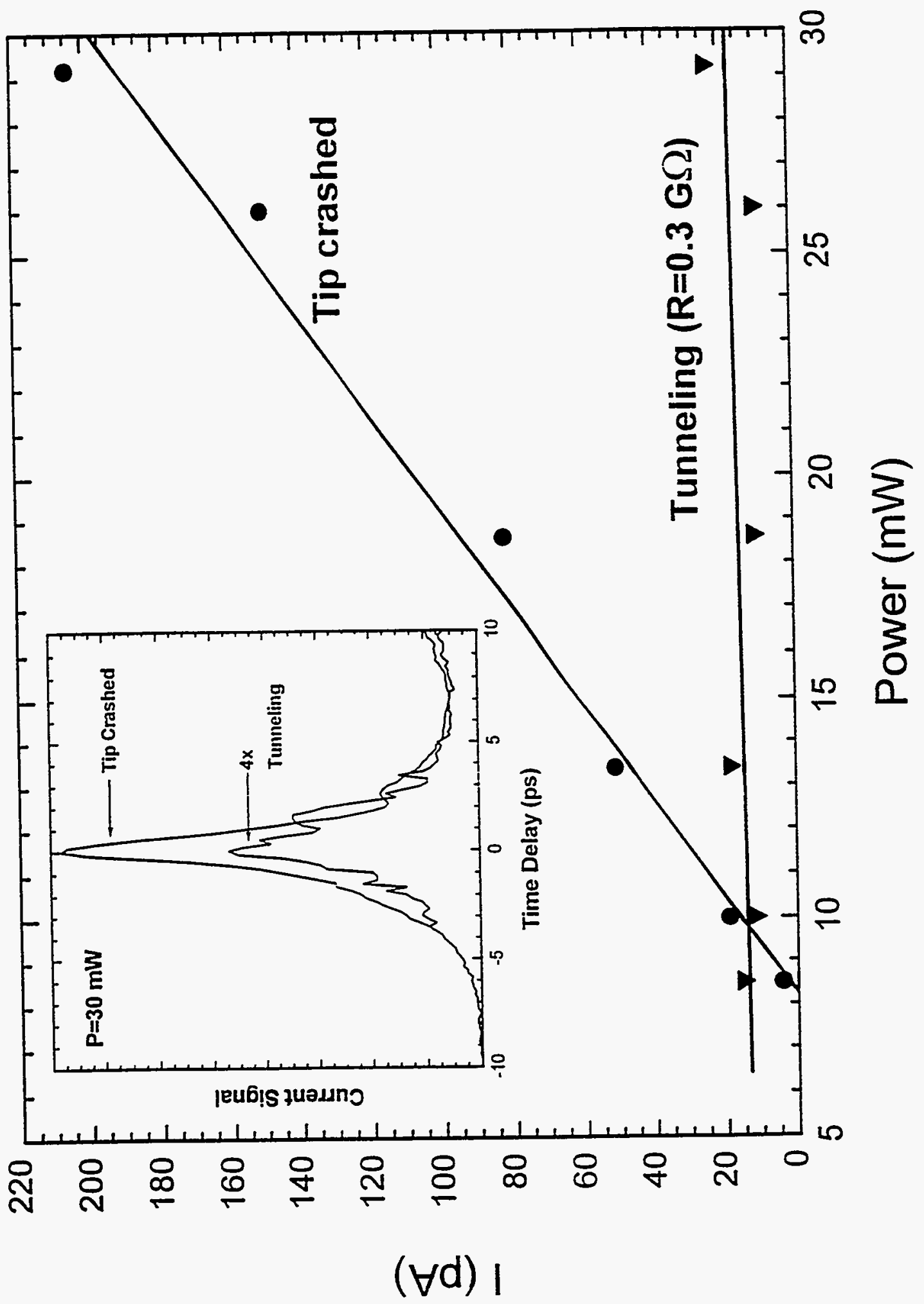

尊 


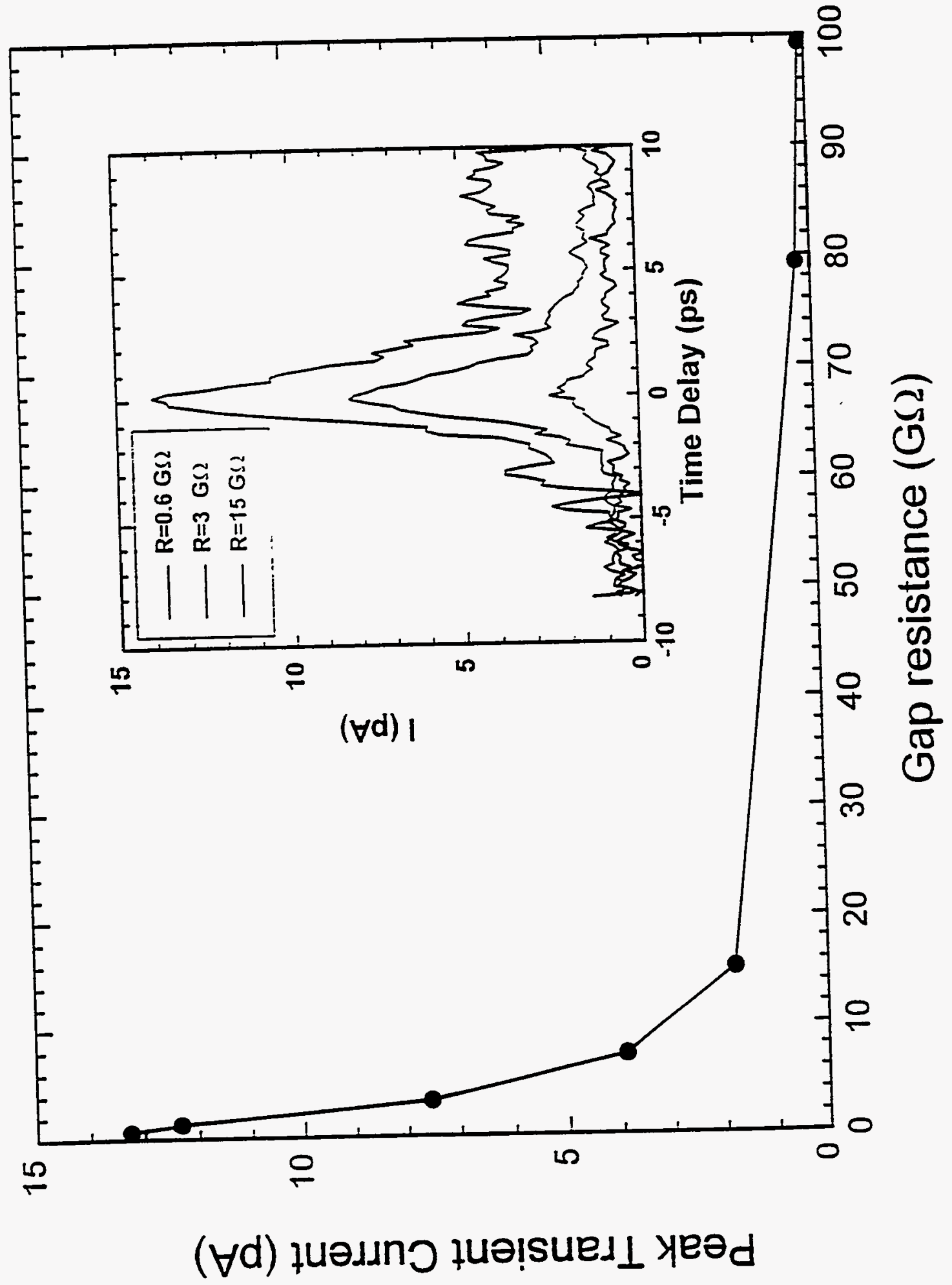

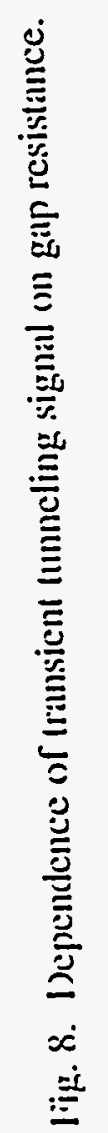



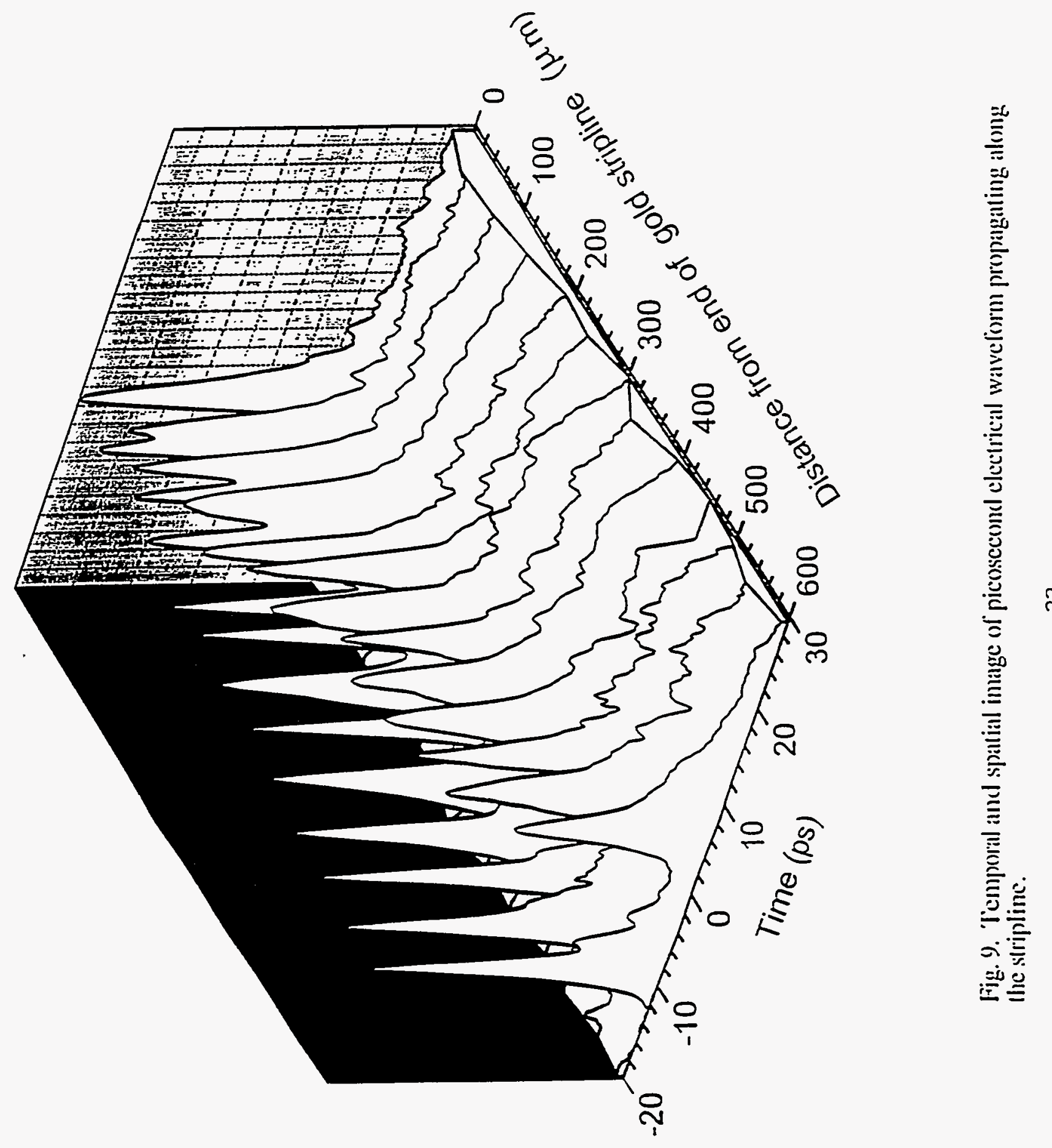

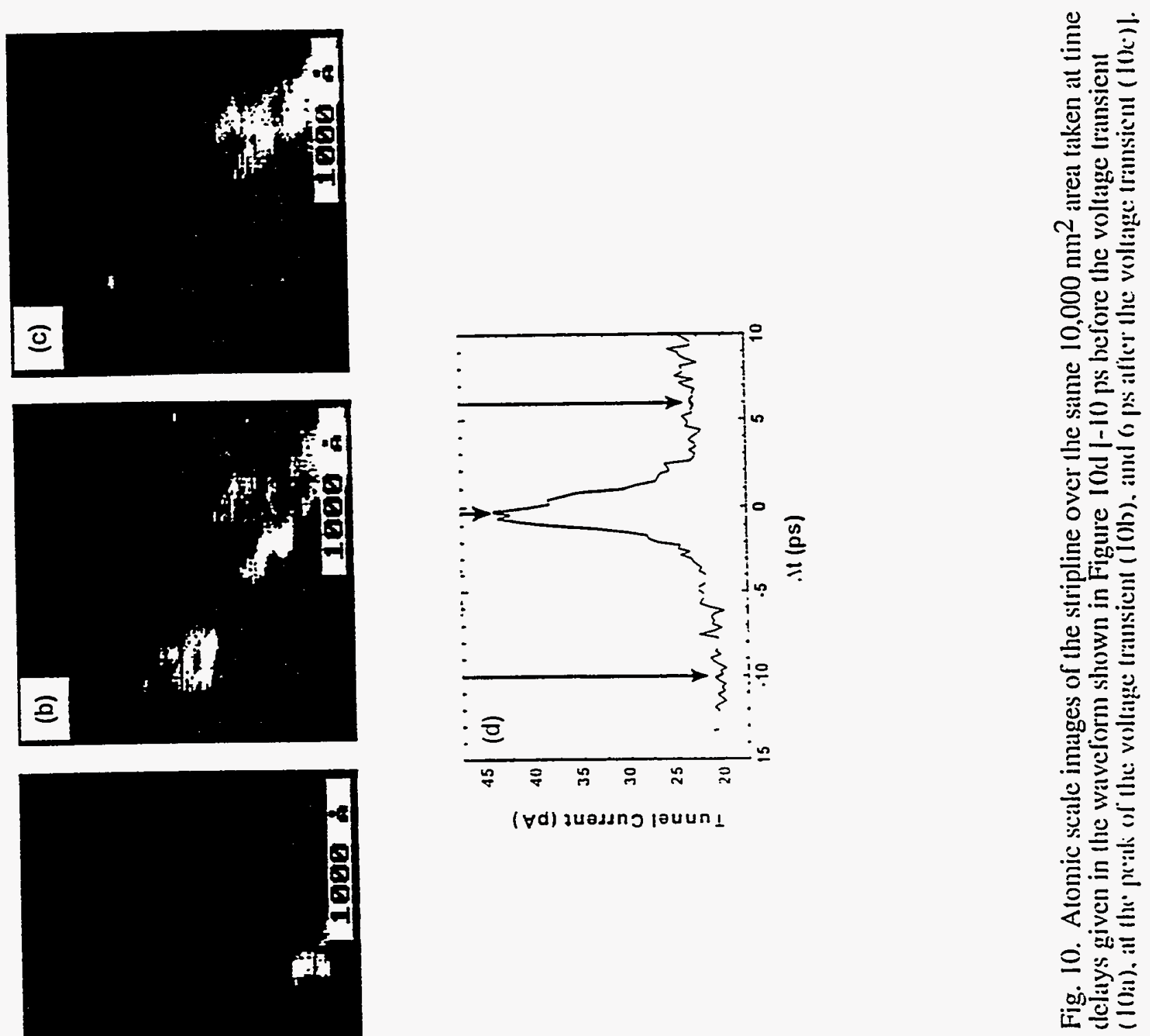
즈 